\title{
ON THE DOUBLE SUSPENSION HOMOMORPHISM
}

BY

\section{MARK MAHOWALD}

ABSTRACT. This paper studies the family of unstable Adams spectral sequences, $E_{2}^{s, t}\left(S^{2 n+1}\right)$. The main results deal with the range of filtrations for which these groups stabilize and for which the groups $E_{2}^{s, t}\left(\Omega^{2} s^{2 n+1}, s^{2 n-1}\right)$ stabilize.

This paper is concerned only with the 2-primary homotopy of spheres and all spaces should be considered as being localized at the prime 2 .

Let $W_{n}$ be the fiber of the map $S^{2 n-1} \rightarrow \Omega^{2} S^{2 n+1}$. Using the unstable Adams spectral sequence theory of Kan et al. [1], we can construct a spectral sequence for $W_{n}$. We wish to normalize so that $E_{2}^{1,2}\left(W_{n}\right)=Z_{2}$ and is the first nonzero group, i.e., $E^{0, t}\left(W_{n}\right)=0$ for all $t$ and $E_{2}^{s, t}\left(W_{n}\right)=0$ for all $s$ if $t=0,1$. Our main result is:

THEOREM 1. There are natural maps

$$
\begin{aligned}
E_{2}^{s, t}\left(W_{1}\right) \stackrel{f_{1}}{\rightarrow} E_{2}^{s, t}\left(W_{2}\right) \stackrel{f_{2}}{\rightarrow} \cdots \stackrel{f_{n-1}}{\longrightarrow} E_{2}^{s, t}(W) \\
\stackrel{f_{n}}{\longrightarrow} \cdots \stackrel{f}{\rightarrow} \operatorname{Ext}_{A}^{s-1, t-1}\left(\tilde{H}^{*}\left(P^{2}\right), Z_{2}\right)
\end{aligned}
$$

so that $f_{n}$ is an isomorphism for $6 s>t+20-4 n$.

In [3], $\operatorname{Ext}_{A}^{s, t}\left(\widetilde{H}^{*}\left(P^{2}\right), Z_{2}\right)$ is calculated for $6 s>t+14$ and the $E_{\infty}$ of the Adams spectral sequence is also obtained for the same range.

This result fits into the general picture in the following way. The Frudenthal suspension theorem says that $\pi_{k}\left(S^{n}\right)$ depends only on $k-n$ for $k<2 n$ - 1. The EHP sequence $\pi_{k}\left(S^{n}\right) \rightarrow \pi_{k+1}\left(S^{n+1}\right) \rightarrow \pi_{j+1}\left(S^{2 n-1}\right)$ and the suspension theorem assert that the amount by which the suspension is not an isomorphism is stable provided $k<4 n-3$. If we think in terms of the Adams spectral sequence then the analog of the Frudenthal result is the following result which is due to Curtis.

TheOREM (CuRTIS). $E_{2}^{s, t}\left(S^{n}\right) \rightarrow E_{2}^{s, t}\left(S^{n+2}\right)$ is an isomorphism for $3 s>$ $t+6-n$ and $n$ odd.

This stability has implications for $\pi_{k}\left(S^{n}\right)$ for arbitrary $k$. An obvious cor-

Received by the editors April 1, 1974.

AMS (MOS) subject classifications (1970). Primary 55E45, 55D40.

Copyright $\odot$ 1975, American Mathematical Society 
ollary of our Theorem 1 is the following analog of the "meta-stable" stability implied by the EHP sequence.

THEOREM 2. The measure of the amount by which $f: E_{2}^{s, t}\left(S^{2 n-1}\right) \rightarrow$ $E_{2}^{s, t}\left(S^{2 n+1}\right)$ fails to be an isomorphism, i.e., $E_{2}^{s, t}\left(\Omega^{2} S^{2 n+1}, S^{2 n-1}\right)$, is independent of $n$ for $6 s>t+20-4 n$.

2. Preliminary definitions. The key tool used in this paper is the $\Lambda$ algebra [1] and [5]. We recall its definition and some of its properties. $\Lambda(n)$ is a graded $Z_{2}$-algebra generated by monomials in $\lambda_{i}, i=0,1, \ldots$, such that if $\lambda_{i_{1}} \lambda_{i_{2}} \ldots \lambda_{i_{j}}$ is a basis element then (1) $i_{1} \leqslant n$, (2) $2 i_{k} \geqslant i_{k+1}$. Such monomials are called admissible. A multiplication is defined by juxtaposition. The following relations are used to change such a product into a sum of admissible monomials. Let $D$ be a derivation with $D \lambda_{i}=\lambda_{i+1}$ and $D^{t}=D\left(D^{t-1}\right)$. Then $D^{t}\left(\lambda_{i} \lambda_{2 i+1}\right)=0$ for all $i$ and $t \geqslant 0$. In $\Lambda(n)$ there is a boundary operator $d$ such that

$$
d \lambda_{n}=\sum_{i=0}^{[n / 2]}\left(\begin{array}{c}
n-i \\
i
\end{array}\right) \lambda_{n-i} \lambda_{i-1} .
$$

We can bigrade $\Lambda(n)$ by assigning filtration $(1, i+1)$ to $\lambda_{i}$. Then $H_{s, t}(\Lambda(n), d)$ $=E_{2}^{s, t}\left(S^{n+1}\right)$.

The EHP sequence produces short exact sequences

$$
0 \rightarrow \Lambda^{s, t}(n-1) \rightarrow \Lambda^{s, t}(n) \rightarrow \Lambda^{s-1, t-n-2}(2 n) \rightarrow 0
$$

where the first is the obvious inclusion and the second map is defined by forgetting $\lambda_{n}$ when it occurs as a leading element in a basis element.

To prove Theorem 1 we wish to look at the double suspension. Let $\Lambda\left(W_{n}\right)=$ $\kappa_{2} \Lambda(4 n) \oplus \kappa_{1} \Lambda(4 n-2)$ and assign $\kappa_{i}$ filtration $(1, i+1)$. Then we have

$$
0 \rightarrow \Lambda^{s, t}(2 n-2) \rightarrow \Lambda^{s, t}(2 n) \stackrel{p}{\rightarrow} \Lambda^{s, t-2 n+2}\left(W_{n}\right) \rightarrow 0
$$

where the first map is the obvious inclusion and the second map satisfies $p\left(\lambda_{2 n} \lambda_{I}\right)$ $=\kappa_{2} \lambda_{I}, p\left(\lambda_{2 n-1} \lambda_{I}\right)=\kappa_{1} \lambda_{I}$ and, if the basis monomial $\lambda_{I}$ starts with $\lambda_{i}$ for $i<$ $2 n-1$, then $p\left(\lambda_{I}\right)=0$. From (2.1) we can define a boundary operator, $d$, in $\Lambda\left(W_{n}\right)$, so that the sequence (2.1) is a short exact sequence of chain complexes. Theorem 1 will be proved explicitly by proving the following.

THEOREM 3. There is a natural sequence of chain maps

$$
\Lambda^{s, t}\left(W_{1}\right) \subset \Lambda^{s, t}\left(W_{2}\right) \subset \cdots \subset \Lambda^{s, t}\left(W_{n}\right) \rightarrow \cdots \rightarrow \kappa_{2} \Lambda \oplus \kappa_{1} \Lambda,
$$

where the last term is associated with $\Lambda\left(P^{2}\right)$ such that $\Lambda\left(W_{n}\right) / \Lambda\left(W_{n+1}\right)$ has zero homology if $6 s>t+14-4 n$, for $n \geqslant 1$. 
3. The chain complex $\Lambda\left(W_{n}\right)$. The first step in proving Theorem 3 is to determine the differential in $\Lambda\left(W_{n}\right)$.

Proposition 3.1. $d\left(\kappa_{2} \lambda_{I} \oplus \kappa_{1} \lambda_{J}\right)=\kappa_{2} d \lambda_{I} \oplus \kappa_{1}\left(\lambda_{0} \lambda_{I}+d \lambda_{J}+a\right)$ where $a=$ 0 if $\lambda_{I} \in \Lambda(4 n-1) \subset \Lambda(4 n)$ and $a=\left(d \lambda_{4 n+1}\right) \lambda_{I^{\prime}}$ if $\lambda_{I}=\lambda_{4 n} \lambda_{I^{\prime}}$.

The proof is long and so we delay it until the end of this section.

Definition 3.2. The map $f_{n}: \Lambda\left(W_{n}\right) \rightarrow \Lambda\left(W_{n+1}\right)$ is given by $f_{n}\left(\kappa_{2} \lambda_{I} \oplus \kappa_{1} \lambda_{J}\right)$ $=\kappa_{2} \lambda_{I} \oplus \kappa_{1}\left(\lambda_{J} \oplus \epsilon \lambda_{4 n+1} \lambda_{I^{\prime}}\right)$ where $\epsilon=0$ if $\lambda_{I} \in \Lambda(4 n-1)$ and $\epsilon=1$ if $\lambda_{I}=$ $\lambda_{4 n} \lambda_{I^{\prime}}$

Proposition 3.3. $f_{n}$ is a chain mapping.

Proof. The proof is clear from 3.1. Indeed,

$$
\begin{aligned}
f\left(d\left(\kappa_{2} \lambda_{I} \oplus \kappa_{1} \lambda_{J}\right)\right) & =f\left(\kappa_{2} d \lambda_{I} \oplus \kappa_{1}\left(d \lambda_{J} \oplus \lambda_{0} \lambda_{I} \oplus \epsilon d \lambda_{4 n+1} \lambda_{I^{\prime}}\right)\right) \\
& =\kappa_{2} d \lambda_{I} \oplus \kappa_{1}\left(\epsilon \lambda_{4 n+1} d \lambda_{I^{\prime}} \oplus d \lambda_{J} \oplus \lambda_{0} \lambda_{I} \oplus \epsilon\left(d \lambda_{4 n+1}\right) \lambda_{I^{\prime}}\right) \\
& =\kappa_{2} d \lambda_{I} \oplus \kappa_{1}\left(d\left(\lambda_{J} \oplus \epsilon \lambda_{4 n+1} \lambda_{I^{\prime}} \oplus \lambda_{0} \lambda_{I}\right)\right. \\
& =d f_{n}\left(\kappa_{2} \lambda_{I} \oplus \kappa_{1} \lambda_{J}\right) .
\end{aligned}
$$

SKETCH OF THE PROOF OF THEOREM 3. We will construct the chain complex $\Lambda\left(W_{n+1} / W_{n}\right)$ and find an artificial complex $\Lambda\left(C_{n}\right)$ which maps into $\Lambda\left(W_{n+1} / W_{n}\right)$. This map will be shown, using an induction hypothesis, to induce an isomorphism. This is done in $\S 4$. Then in $\S 5$ we look at an $A_{1}$ free stable complex $X$ and show that $\Lambda\left(C_{n}\right)$ maps into $\Lambda(X)$ and also induces an isomorphism in an appropriate range of dimensions. Finally, we recall that $\operatorname{Ext}_{A}^{s, t}\left(A_{1}, Z_{2}\right)=0$ for a certain range of dimensions and this completes the proof.

Proof of Proposition 3.1. We need to calculate the differential in $\kappa_{2} \Lambda(4 n) \oplus \kappa_{1} \Lambda(4 n-2)$. The differential is evaluated by the following sequence of maps:

$$
\kappa_{2} \Lambda(4 n) \oplus \kappa_{1} \Lambda(4 n-2) \rightarrow \Lambda(2 n) \stackrel{d}{\rightarrow} \Lambda(2 n) \rightarrow \kappa_{2} \Lambda(4 n) \oplus \kappa_{1} \Lambda(4 n-2)
$$

where the first map is given by $\kappa_{i} \rightarrow \lambda_{2 n+i-2}$ and the last map is $p$ of 3.1. Thus we need to put in admissible form $\left(d \lambda_{2 n}\right) \lambda_{I}$ and $\left(d \lambda_{2 n+1}\right) \lambda_{J}$ and determine the coefficient of $\lambda_{2 n-1}$. We need the following lemma.

LEMMA 3.5. $\lambda_{i} \Lambda(k) \subset \Lambda(k-i-1) \cup \Lambda(i)$.

Proof. We wish to look at $\lambda_{i} \lambda_{J}$ where $\lambda_{J} \in \Lambda(k)$. If $\lambda_{J}$ is also in $\Lambda(2 i)$ then $\lambda_{i} \lambda_{J}$ is admissible and in $\Lambda(i)$. We suppose that $\lambda_{i} \lambda_{J}$ is not admissible as it stands. If $|J|=1$ then $\lambda_{i} \lambda_{j}=\Sigma_{k \geqslant 2 i+1} a_{k} \lambda_{i+j-k} \lambda_{k}$ and this is in $\Lambda(j-i-1)$. Suppose we have established the lemma for all $i$ and $J$ such that $|J|<n$. Suppose $j_{1}=2 l$. Then 


$$
\begin{aligned}
\lambda_{l-1} \lambda_{J} & =\lambda_{l-1} \lambda_{j_{1}} \lambda_{J^{\prime}}=\lambda_{l} \lambda_{j_{1}-1} \lambda_{J^{\prime}} \\
& \in \lambda_{l} \lambda_{j_{1}-1} \Lambda^{n-1}\left(2 j_{1}\right) \subset \lambda_{l} \Lambda^{n}\left(j_{1}\right) \subset \Lambda^{n+1}(l)
\end{aligned}
$$

and this is the lemma. Suppose $j_{1}=2 l+1$. Then $\lambda_{l-1} \lambda_{j_{1}} \lambda_{J^{\prime}}=\lambda_{l+1} \lambda_{j_{1}-2} \lambda_{J^{\prime}} \in$ $\lambda_{l+1} \Lambda^{n}\left(j_{1}+1\right) \in \Lambda(l+1)$ which is the lemma. Now suppose we have established the lemma for $\lambda_{\bar{i}} \lambda_{\bar{J}}$ if $|\bar{J}|<n$ or if $|\bar{J}|=n$ then $\bar{j}_{1}-2 \bar{i}-1<q$. Suppose $|J|=n$ and $j_{1}-2 i-1=q$. Then

$$
\lambda_{i} \lambda_{j}=\sum_{j_{1}>k>2 i+1} a_{k} \lambda_{i+j_{1}-k} \lambda_{k} \lambda_{J^{\prime}}+\lambda_{j_{1}-i-1} \lambda_{2 i+1} \lambda_{J^{\prime}}
$$

If $j_{1} \geqslant k>2 i+1$ then

$$
\lambda_{i+j_{1}-k} \lambda_{k} \Lambda^{n-1}\left(2 j_{1}\right) \subset \lambda_{i+j_{1}-k} \Lambda^{n}\left(2 j_{1}-k-1\right) .
$$

Since $2 j_{1}-k-1-2\left(i+j_{1}-k\right)-1<q$ the induction hypothesis implies that the last expression is in $\Lambda^{n+1}\left(j_{1}-i-2\right)$. Finally

$$
\lambda_{j_{1}-i-1} \lambda_{2 i+1} \Lambda^{n-1}\left(2 j_{1}\right) \subset \lambda_{j_{1}-i-1} \Lambda\left(2 j_{1}-2 i-2\right) \subset \Lambda\left(j_{1}-i-1\right) .
$$

This completes the double induction and the proof of the lemma.

Now we can compute the coefficient of $\lambda_{2 n-1}$ in $\left(d \lambda_{2 n}\right) \lambda_{I}$. If $I$ begins with $i_{1}<$ $4 n$ then

$$
\begin{aligned}
d\left(\lambda_{2 n}\right) \lambda_{I}= & \sum_{i>1}\left(\begin{array}{c}
2 n-i \\
i
\end{array}\right) \lambda_{2 n-i} \lambda_{i-1} \lambda_{I} \lambda_{2 n-1} \lambda_{0} \lambda_{I} \\
& +\sum_{i>1}\left(\begin{array}{c}
2 n-i \\
i
\end{array}\right) \lambda_{2 n-i} \lambda_{i-1} \lambda_{I}
\end{aligned}
$$

and

$$
\lambda_{2 n-i} \lambda_{i-1} \Lambda(4 n-1) \subset \lambda_{2 n-1} \Lambda(4 n-i-1) \subset \Lambda(2 n-2)
$$

Thus if $\lambda_{I} \in \Lambda(4 n-1), d\left(\kappa_{2} \lambda_{I}\right)=\kappa_{2} d \lambda_{I}+\kappa_{1} \lambda_{0} \lambda_{I}$.

Using 3.5 in a similar way, we see that if $i_{1}=4 n$ then

$$
\begin{aligned}
\left(d \lambda_{2 n}\right) \lambda_{I} & =\sum_{i \geqslant 1}\left(\begin{array}{c}
2 n-i \\
i
\end{array}\right) \lambda_{2 n-i} \lambda_{i-1} \lambda_{4 n} \lambda_{I^{\prime}} \\
& =\sum_{i>1}\left(\begin{array}{c}
2 n-i \\
i
\end{array}\right) \lambda_{2 n-1} \lambda_{4 n-2 i+1} \lambda_{2 i-1} \lambda_{I^{\prime}}+c+\lambda_{2 n-1} \lambda_{0} \lambda_{I}
\end{aligned}
$$

where $c \in \Lambda(2 n-2)$.

LEMMA 3.6.

$$
d \lambda_{4 n+1}=\sum_{i>1}\left(\begin{array}{c}
2 n-i \\
i
\end{array}\right) \lambda_{4 n-2 i+1} \lambda_{2 i-1}
$$


Proof.

$$
d \lambda_{4 n+1}=\sum_{j \geqslant 1}\left(\begin{array}{c}
4 n+1-j \\
j
\end{array}\right) \lambda_{4 n-j} \lambda_{j-1} .
$$

Thus we need to show that $\left(4_{j}^{4 n+1-j}\right) \equiv 0(\bmod 2)$ if $j \equiv 1(\bmod 2)$ and $\left(\begin{array}{c}4 n+1-2 i \\ 2 i\end{array}\right) \equiv\left({ }^{2 n-i} i\right) \bmod 2$. Note that if $\alpha$ generates $H^{1}(R P)$ then $S q^{j} \alpha^{4 n+1-j}=\left({ }^{4 n+1-j}\right) \alpha^{4 n+1}$ and if $\kappa$ generates $H^{2}(C P)$ then $S q^{2 i} \kappa^{2 n-i}=$ $\left(\begin{array}{c}2 n-i \\ i\end{array}\right) \kappa^{2 n}$. Since $\alpha^{4 n+1} \notin \operatorname{im} S q^{1}\left({ }^{4 n+1-j}\right) \equiv 0$ (2) for $j \equiv 1$ (2). Since there is a stable map $f: \Sigma C P \rightarrow R P$ so that $f^{*}\left(\alpha^{2 i+1}\right)=\kappa^{i}$ we see that

$$
\left(\begin{array}{c}
4 n+1-2 i \\
2 i
\end{array}\right) \equiv\left(\begin{array}{c}
2 n-i \\
i
\end{array}\right) \bmod 2
$$

We return to the proof of 3.1. Note that $\lambda_{2 n-1} \lambda_{4 n-1} \lambda_{1} \lambda_{I^{\prime}}=0$ since $2(2 n-1)$ $+1=4 n-1$. Thus

$$
\sum_{i \geq 2}\left(\begin{array}{c}
2 n-i \\
i
\end{array}\right) \lambda_{2 n-1} \lambda_{4 n-2 i+1} \lambda_{2 i-1} \lambda_{I^{\prime}}=\lambda_{2 n-1}\left(d \lambda_{4 n+1}\right) \lambda_{I^{\prime}}
$$

by 4.3. Hence $d\left(\lambda_{2 n}\right) \lambda_{I}=\lambda_{2 n-1}\left(\lambda_{0} \lambda_{I}+\left(d \lambda_{4 n+1}\right) \lambda_{I^{\prime}}\right)+c^{\prime}$ where $c^{\prime} \in$ $\Lambda(2 n-2)$. Putting all of this together, we see that $d\left(\lambda_{2 n} \lambda_{I}+\lambda_{2 n-1} \lambda_{J}\right)=$ $\lambda_{2 n-1}\left(d \lambda_{J}+\lambda_{0} \lambda_{I}+a\right)+\lambda_{2 n} d \lambda_{I}+c^{\prime \prime}$ where $c^{\prime \prime} \in \Lambda(2 n-2)$ and this proves the proposition.

4. The chain complex $\Lambda\left(F_{n}\right)=\Lambda\left(W_{n+1} / W_{n}\right)$. Let $\Lambda\left(F_{n}\right)$ be the quotient chain complex of the map $f_{n}$. Then $\Lambda\left(F_{n}\right)=\kappa_{2} \bigoplus_{i=1}^{4} \lambda_{4 n+i} \Lambda(8 n+2 i) \oplus$ $\kappa_{1} \bigoplus_{i=-1}^{2} \lambda_{4 n+1} \Lambda(8 n+2 i)$ and $\Lambda\left(F_{n}\right)$ receives a differential from $\Lambda\left(W_{n+1}\right)$. The differential is calculated by the following composite $\Lambda\left(F_{n}\right) \stackrel{i}{\rightarrow} \Lambda\left(W_{n+1}\right) \stackrel{d}{\rightarrow}$ $\Lambda\left(W_{n+1}\right) \stackrel{p}{\rightarrow} \Lambda\left(F_{n}\right)$ where $i$ is the vector space inclusion and $p$ is the projection. The exact form of this differential is very complicated and we will not need it.

Let

$$
\begin{aligned}
\Lambda\left(C_{n}\right)= & \kappa_{2} \oplus\left[\left(\lambda_{4 n+1} \oplus \lambda_{4 n+3}\right) \Lambda(8 n-2) \oplus\left(\lambda_{4 n+2}+\lambda_{4 n+4}\right) \Lambda(8 n)\right] \\
& +\kappa_{1} \oplus\left[\left(\lambda_{4 n-1} \oplus \lambda_{4 n+1}\right) \Lambda(8 n-2) \oplus\left(\lambda_{4 n}+\lambda_{4 n+2}\right) \Lambda(8 n)\right] .
\end{aligned}
$$

Let $g: \Lambda\left(C_{n}\right) \rightarrow \Lambda\left(F_{n}\right)$ be given by

$$
g\left(\kappa_{i}\left(\lambda_{4 n+2 j} \lambda_{I}+\lambda_{4 n+2 j-1} \lambda_{J}\right)\right)=\kappa_{i}\left(\lambda_{4 n+2 j} \lambda_{I} \lambda_{4 n+2 j-1}\left(\lambda_{8 n+1} \lambda_{I^{\prime}}+\lambda_{J}\right)\right)
$$

for $j=1,2, i=2$ and $j=1, i=1$ and where $\lambda_{I^{\prime}}$ is zero unless $\lambda_{I}=\lambda_{8 n^{\prime}} \lambda_{I^{\prime}}$; $\lambda_{I} \in \Lambda(8 n)$ and $\lambda_{J} \in \Lambda(8 n-2)$;

$$
g\left(\kappa_{1}\left(\lambda_{4 n} \lambda_{J}+\lambda_{4 n-1} \lambda_{I}\right)\right)=\kappa_{1}\left(\lambda_{4 n} \lambda_{J}+\lambda_{4 n-1} \lambda_{I}\right) .
$$

Lemma 4.1. $d g \subset \operatorname{im} g$. 
Proof. The $d$ in $\Lambda\left(F_{n}\right)$ is calculated by retracting $\Lambda\left(F_{n}\right)$ into $\Lambda\left(W_{n+1}\right)$, calculating $d$ in $\Lambda\left(W_{n+1}\right)$ and projecting back to $\Lambda\left(F_{n}\right)$. When this is done for the image of $g$ we get the following formulae:

$$
\begin{aligned}
d g \kappa_{2} \lambda_{4 n+4} \lambda_{I}=\kappa_{2} & \lambda_{4 n+4} d \lambda_{I}+\lambda_{4 n+3}\left(\lambda_{0} \lambda_{I}+\left(d \lambda_{8 n+1}\right) \lambda_{I^{\prime}}\right. \\
& \left.\left.+\lambda_{8 n+1} d \lambda_{I^{\prime}}\right)+\lambda_{n+2} \lambda_{1} \lambda_{I}\right) \\
& +\kappa_{1}\left(\lambda_{4 n+2}\left(\lambda_{2} \lambda_{I}+\lambda_{1} \lambda_{8 n+1} \lambda_{I^{\prime}}\right)+\epsilon_{n} \lambda_{4 n+1} \lambda_{3} \lambda_{I}\right. \\
& \left.+\lambda_{4 n}\left(\lambda_{4} \lambda_{I}+\lambda_{3} \lambda_{8 n+1} \lambda_{I^{\prime}}\right)\right)
\end{aligned}
$$

where $\epsilon_{n} \equiv(m) \bmod 2$ and where $\lambda_{I^{\prime}}=0$ unless $\lambda_{I}=\lambda_{8 n} \lambda_{I^{\prime}}$.

$d\left(g \kappa_{2} \lambda_{4 n+3} \lambda_{J}\right)=\kappa_{1}\left(\lambda_{4 n+2} \lambda_{1}+\lambda_{4 n} \lambda_{3}\right) \lambda_{J}+\kappa_{2} \lambda_{4 n+3} d \lambda_{J}$,

$d\left(g_{2} \lambda_{4 n+2} \lambda_{I}\right)=\kappa_{2}\left(\lambda_{4 n+2} d \lambda_{I}+\lambda_{4 n+1}\left(\lambda_{0} \lambda_{I}+\left(d \lambda_{8 n+1}\right) \lambda_{I^{\prime}}+\lambda_{8 n+1} d \lambda_{I^{\prime}}\right)\right)$

$+\kappa_{1}\left(\lambda_{4 n+1} \lambda_{1} \lambda_{I}+\lambda_{4 n}\left(\lambda_{2} \lambda_{I}+\lambda_{1} \lambda_{8 n+1} \lambda_{I^{\prime}}\right)+\lambda_{4 n-1} \lambda_{3} \lambda_{I}\right)$,

$d\left(g_{2} \lambda_{4 n+1} \lambda_{J}\right)=\kappa_{2} \lambda_{4 n+1} d \lambda_{J}+\kappa_{1} \lambda_{4 n} \lambda_{1} \lambda_{J}$,

$d\left(g_{1} \lambda_{4 n+2} \lambda_{I}\right)=\kappa_{1}\left(\lambda_{4 n+1}\left(\lambda_{0} \lambda_{I}+d\left(\lambda_{8 n+1}\right) \lambda_{I^{\prime}}\right)+\lambda_{4 n-1} \lambda_{2} \lambda_{I}+\lambda_{4 n+2} d \lambda_{I}\right)$

$$
+\lambda_{4 n-1}\left(\lambda_{1} \lambda_{8 n+1}+d \lambda_{8 n+3}\right) \lambda_{I^{\prime}} \text {, }
$$

$d\left(g \kappa_{1} \lambda_{4 n+1} \lambda_{J}\right)=\kappa_{1}\left(\lambda_{4 n+1} d \lambda_{J}+\lambda_{4 n-1} \lambda_{1} \lambda_{J}\right)$,

$$
d\left(g_{1} \lambda_{4 n} \lambda_{I}\right)=\kappa_{1}\left(\lambda_{4 n} d \lambda_{I}+\lambda_{4 n-1}\left(\lambda_{0} \lambda_{I}+d \lambda_{8 n+1} \lambda_{I^{\prime}}\right)\right) \text {, }
$$

$d\left(\kappa_{1} \lambda_{4 n-1} \lambda_{J}\right)=\kappa_{1} \lambda_{4 n-1} d \lambda_{J}$.

To see this observe that $d\left(\kappa_{j} \lambda_{4 n+i}\right) \lambda_{I}$ in $\Lambda\left(W_{n+1}\right)$ involves terms of the form $\kappa_{j} \lambda_{4 n-p} \lambda_{p+i-1} \lambda_{I}$ or, if $j=2$, terms like $\kappa_{j-1} \lambda_{4 n-p} \lambda_{p+i} \lambda_{I}$. If $j \neq 1$ and $i$ is neither 0 nor 2 , terms such as these, when made admissible, project to zero in $\Lambda\left(E_{n}\right)$. Indeed, $\lambda_{4 n-p} \lambda_{p+i} \lambda_{I} \subset \Lambda(4 n-i-1) \cup \Lambda(4 n-p) \subset \Lambda(4 n-2)$ except for the above exceptions. In the case of exceptions when $i=0$ the argument is just that of $\S 3$. When $i=2$ we see that

$$
p \kappa_{1} d \lambda_{4 n+1} \lambda_{8 n+1}=\sum\left(\begin{array}{c}
4 n+1-i \\
i
\end{array}\right) k_{1} \lambda_{4 n-1} \lambda_{8 n-2 i+3} \lambda_{2 i-1} .
$$

Also $d \lambda_{8 n+3}=\Sigma\left({ }^{8 n+3-i}\right) \lambda_{8 n+3-i} \lambda_{i-1}$. The argument from Lemma 3.5 shows that these are the same and thus $p k_{1} d\left(\lambda_{4 n+1}\right) \lambda_{8 n+1}=$ $\kappa_{1} \lambda_{4 n-1}\left(\lambda_{1} \lambda_{8 n+1}+d\left(\lambda_{8 n+3}\right)\right)$. Thus we see that in all cases the above formulae describe what happens.

It is a simple direct verification now that $d g \subset \operatorname{im} g$. We will do the first one term by term. Suppose that $\lambda_{I}=\lambda_{8 n} \lambda_{I^{\prime}}$. The other case is easier. Consider 
$\kappa_{2}\left(\lambda_{4 n+4}\left(d \lambda_{8 n}\right) \lambda_{I^{\prime}}+\lambda_{8 n} d \lambda_{I^{\prime}}\right)+\lambda_{4 n+3}\left(\lambda_{0} \lambda_{8 n} \lambda_{I^{\prime}}+d \lambda_{8 n+1} \lambda_{I^{\prime}}+\lambda_{8 n+1} d \lambda_{I^{\prime}}\right)$.

The classes $\left(d \lambda_{8 n}\right) \lambda_{I^{\prime}} \subset \Lambda(8 n-1)$ by (3.5). The class

$$
\lambda_{0} \lambda_{8 n^{\prime}} \lambda_{I^{\prime}}+\left(d \lambda_{8 n+1}\right) \lambda_{I^{\prime}} \subset \Lambda(8 n-2)
$$

by (3.4). Thus the above term is $\left.g\left(\kappa_{2} \lambda_{4 n+4}\left(a+\lambda_{8 n} d \lambda_{I^{\prime}}\right)+\lambda_{4 n+3}(b)\right)\right)$ where $a \in \Lambda(8 n-1)$ and $b \in \Lambda(8 n-2)$. The class $\kappa_{2} \lambda_{4 n+2} \lambda_{1} \lambda_{I}$ is handled by noting that $\lambda_{1} \lambda_{I} \subset \Lambda(8 n-2) \sqsubset \Lambda(8 n)$. Continuing with the terms of $d g \kappa_{2} \lambda_{4 n+4} \lambda_{I}$ we see $\lambda_{2} \lambda_{I} \subset \Lambda(8 n-3) \subset \Lambda(8 n) ; \lambda_{1} \lambda_{8 n+1} \lambda_{I^{\prime}} \subset \lambda_{1} \Lambda(8 n+1) \subset \Lambda(8 n-1)$; $\lambda_{3} \lambda_{I} \subset \Lambda(8 n-4) \subset \Lambda(8 n-2) ; \lambda_{4} \lambda_{I} \subset \Lambda(8 n-5) \subset \Lambda(8 n) ; \lambda_{3} \Lambda(8 n+1) \subset$ $\Lambda(8 n-3) \subset \Lambda(8 n)$. All the other cases are similarly handled. This proves the lemma.

A key step in the proof of 2.2 is the following result.

Lemma 4.2. For a fixed $t$, if Theorem 3 is true for all $t^{\prime}<t$, then $g$ induces an isomorphism in homology for $6 s>t+3-12 n$.

Proof. We will filter the map $g$ in the following fashion.

$$
\begin{aligned}
& A_{1}=\kappa_{1}\left(\lambda_{4 n}{ }_{1} \Lambda(8 n-2) \oplus \lambda_{4 n} \Lambda(8 n)\right) \stackrel{g}{\rightarrow} \kappa_{1}\left(\lambda_{4 n-1} \Lambda(8 n-2) \oplus \lambda_{4 n} \Lambda(8 n)\right)=B_{1}, \\
& n \quad{ }^{n}=A_{1} \oplus \kappa_{1}\left(\lambda_{4 n+1} \Lambda(8 n-2) \oplus \lambda_{4 n+2} \Lambda(8 n)\right) \rightarrow \kappa_{1} \bigoplus_{i=-1}^{2} \lambda_{4 n+i} \Lambda(8 n+2 i)=B_{2}, \\
& A_{3}=A_{2} \oplus \kappa_{2}\left(\lambda_{4 n+1} \Lambda(8 n-2) \oplus \lambda_{4 n+2} \Lambda(8 n)\right) \\
& \quad \rightarrow B_{2} \oplus \kappa_{2} \bigoplus_{i=1} \lambda_{4 n+i} \Lambda(8 n+2 i)=B_{3}, \\
& A_{4}=\Lambda\left(C_{n}\right) \rightarrow \Lambda\left(F_{n}\right)=B_{4} .
\end{aligned}
$$

For the resulting spectral sequence we see that $E_{0}^{s, t, i}(C)=\left(A_{i+1} / A_{i}\right)^{s, t}=$

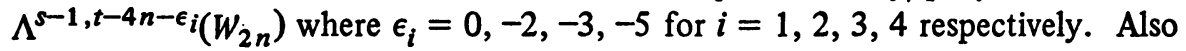
$E_{0}^{s, t, i}(F)=\left(B_{i+1} / B_{i}\right)^{s, t}=\Lambda^{s-1, t-4 n-\epsilon_{i}}\left(W_{2 n+\delta_{i}}\right)$ where $\epsilon_{i}$ is as above and $\delta_{i}=$ $0,1,1,2$ for $i=1,2,3,4$ respectively. The map $g$ induces

$$
g_{i}: E_{0}^{s, t, i}(C) \rightarrow E_{0}^{s, t, i}(F)
$$

and $g_{1}$ is an isomorphism, $g_{2}$ and $g_{3}$ are $f_{2 n}$ and $g_{4}$ is $f_{2 n+1} \circ f_{2 n}$. These are quite easily seen but let us look at $g_{4}$.

$$
\begin{aligned}
g_{4}\left(\kappa_{2} \lambda_{4 n+3} a+\lambda_{4 n+4} \lambda_{8 n+8} b+\lambda_{4 n+4} c\right) \\
=\kappa_{2}\left(\lambda_{4 n+3} a+\lambda_{4 n+4} \lambda_{8 n+8}+\lambda_{4 n+3} \lambda_{8 n+9} b+\lambda_{4 n+4} c\right)
\end{aligned}
$$

and this is just what $f_{2 n}$ does. The second inclusion is just the identity.

If Theorem 3 is true for $t^{\prime}<t$, then $g$ induces an isomorphism at the $E_{1}$ 
level, $g_{\#} E_{1}^{s, t, i}(C) \simeq E_{1}^{s, t, i}(F)$ for all $i$ if $6 s>t-12 n+24$. Thus $g_{\infty}$ is an isomorphism for all $i$, if $6 s>t-12 n+30$.

This proves the lemma.

5. A second auxilary complex. The complex $\Lambda\left(C_{n}\right)$ does not represent anything geometric that has been identified. It was introduced because it also is comparable with an identifiable stable complex.

The following is an easy exercise in stable homotopy.

Proposition 5.1. Let $A_{1}$ be the subalgebra of $A$, the Steenrod algebra, generated by $S q^{1}$ and $S q^{2}$. There is a space $X$ such that $H^{*}(X)$ is a free module over $A_{1}$ on one generator $x$.

Proof. Take $K\left(Z_{2}, n\right)$ for $n \geqslant 6$ and kill $S q^{4}, S q^{4} S q^{2}$ and everything in dimension above $n+6$. The resulting space is $X$.

There is a choice of $X$ so that $S q^{4} S q^{2} x=0$ and one in which $S q^{4} S q^{2} x=$ $S q^{3} S q^{3} x$. Let $X_{1}$ have $S q^{4} S q^{2} x \neq 0$ and $X_{2}$ have $S q^{4} S q^{2} x=0$. In both $X_{k}$ we require $S q^{6} x=0$.

Proposition 5.2. $\Lambda\left(X_{k}\right)=\bigoplus_{i=1}^{2} \bigoplus_{j=2 i-3}^{2 i} \kappa_{i, j} \Lambda$ with

$$
\begin{aligned}
& d\left(\kappa_{2,4}\right)=\kappa_{2,3} \lambda_{0}+\kappa_{2,2} \lambda_{1}+\kappa_{1,2} \lambda_{2}+(k)_{\bmod 2} \kappa_{1,1} \lambda_{3}+\kappa_{1,0} \lambda_{4}, \\
& d\left(\kappa_{2,3}\right)=\kappa_{1,2} \lambda_{1}+\kappa_{1,0} \lambda_{3}, \\
& d\left(\kappa_{2,2}\right)=\kappa_{2,1} \lambda_{0}+\kappa_{1,1} \lambda_{1}+\kappa_{1,0} \lambda_{2}+\kappa_{1,-1} \lambda_{3}, \\
& d\left(\kappa_{2,1}\right)=\kappa_{1,0} \lambda_{1}, \\
& d\left(\kappa_{1,2}\right)=\kappa_{1,1} \lambda_{0}+\kappa_{1,-1} \lambda_{2}, \\
& d\left(\kappa_{1,1}\right)=\kappa_{1,-1} \lambda_{1}, \\
& d\left(\kappa_{1,0}\right)=\kappa_{1,-1} \lambda_{0} .
\end{aligned}
$$

Proof. The L.C.S. $E_{1}$ term for a stable complex is given by $H_{*}\left(X ; Z_{2}\right) \otimes$ $\Lambda$ and the differential is given by $d(a \otimes 1)=\Sigma_{i} S q_{*}^{i} a \otimes \lambda_{i-1}$ where $S q_{*}^{i}$ : $H_{j}\left(X ; Z_{2}\right) \rightarrow H_{j-i}\left(X ; Z_{2}\right)$ is the dual Steenrod square. A direct check of the squaring operations in $A_{1}$ gives the result. The following picture may help the reader. Each 0 represents a cell and $0-0$ represents $S q^{1}$ and 00 represents $S q^{2}$. Note that there are several other $S q^{4}$ 's nonzero in the complex. Since $S q^{5}+$ $S q^{4} S q^{1}=S q^{2} S q^{3}$ and $S q^{2} S q^{3} \neq 0$ and $S q^{1} S q^{4} x=0$, we see that $S q^{4} S q^{1} x \neq$ 0. Since $S q^{6}=S q^{5} S q^{1}+S q^{2} S q^{4}$ and $S q^{5} S q^{1} \neq 0$ we see that $S q^{6}=0 \mathrm{im}$ plies $S q^{4} x \neq 0$. These are reflected in the differentials given above.

Let $\bar{g}: \Lambda\left(C_{n}\right) \rightarrow \Lambda\left(X_{(n)}\right)$, where $(n)$ is the congruence class of $n \bmod 2$, be given by: 


$$
\begin{aligned}
& \bar{g}_{\kappa_{j}} \lambda_{4 n+2 i} \lambda_{I}=\kappa_{j, 2 i} \lambda_{I}+\kappa_{2,2 i-1} \lambda_{8 n+1} \lambda_{I^{\prime}}, \quad j=2, i=1,2 ; j=1, i=0, \\
& \bar{g}_{\kappa_{1}} \lambda_{4 n+2} \lambda_{I}=\kappa_{1,2} \lambda_{I}+\kappa_{1,1} \lambda_{8 n+1} \lambda_{I^{\prime}}+\kappa_{1,-1} \lambda_{8 n+3} \lambda_{I^{\prime}},
\end{aligned}
$$

where $\lambda_{I^{\prime}}=0$ unless $\lambda_{I}=\lambda_{8 n} \lambda_{I^{\prime}} ;$ and $\bar{g}_{\kappa_{j}} \lambda_{4 n+2 i-1} \lambda_{I}=\kappa_{j, 2 i-1} \lambda_{I}$.

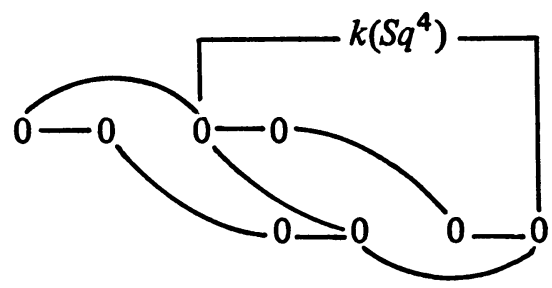

FIGURE 1. $\widetilde{H}^{*}(X)$

Proposition 5.3. $\bar{g}$ is a chain map.

Proof. This is a direct comparison of the two sets of formulae.

Analogously to Lemma 4.2 we have

LEMMA 5.4. For a fixed $t$, if Theorem 3 is true for $t^{\prime}<t$, then $\bar{g}$ induces an isomorphism in homology for $6 s>t+3-12 n$.

The proof follows closely to that of Lemma 4.2.

6. Proof of Theorem 3. The last step in the proof of Theorem 3 is the following:

Proposition 6.1 [3, Corollary 4]. $H_{s, t}\left(\Lambda\left(X_{(n)}, d\right)\right)=0$ if $6 s>t-4 n$ +14 .

Now the proof of Theorem 3 follows easily. First note that Theorem 3 is true if $t=1$. Then note that the case $n=1$ is not needed in the induction and thus

$$
\{(s, t) ; 6 s>t+30-12 n\} \supset\{(s, t) ; 6 s>t-14 n+14\} \quad \text { if } n>1 .
$$

The first is when $H_{s, t}\left(\Lambda\left(X_{(n)}, d\right)\right) \simeq E_{2}^{s, t}\left(F_{n}\right)$ (4.2 and 5.4) and the second is when the left-hand side is isomorphic to zero (6.1).

\section{REFERENCES}

1. A. Bousfield, E. B. Curtis, D. M. Kan, D. G. Quillen, P. L. Rector and J. W. Schlesinger, The mod-p lower central series and the Adams spectral sequence, Topology 5 (1966), 331-342. MR 33 \#8002.

2. Mark Mahowald, The metastable homotopy of $S^{n}$, Mem. Amer. Math. Soc. No. 72 (1967). MR 38 \#5216. 
3. Mark Mahowald, The order of the image of the J-homomorphism, Bull. Amer. Math. Soc. 76 (1970), 1310-1313.

4. H. Toda, Order of the identity class of a suspension space, Ann. of Math. (2) 78 (1963), 300-325. MR 27 \#6271.

5. E. B. Curtis, Simplicial homotopy theory, Advances in Math. 6 (1971), 107-209. MR 43 \#5529.

DEPARTMENT OF MATHEMATICS, NORTHWESTERN UNIVERSITY, EVANSTON, ILLINOIS 60201 